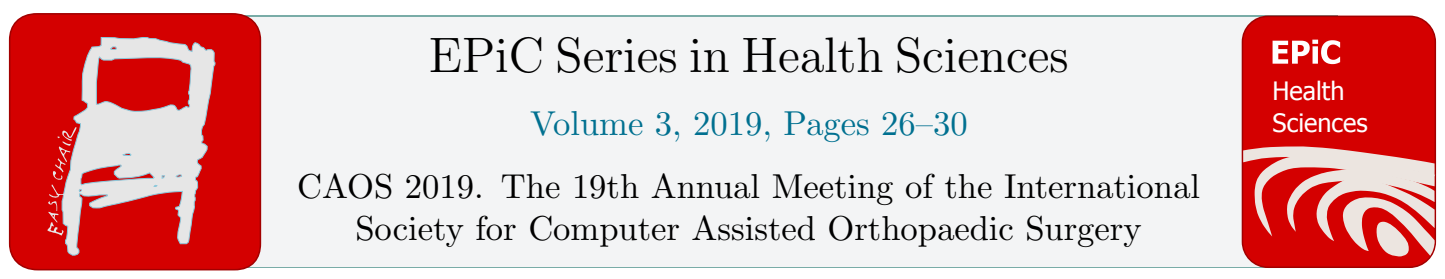

\title{
Relationship of the tibial tuberosity (TT) position, tibial tuberosity-trochlear groove (TT- TG) distance, and internal and external rotation of the knee under weight-bearing conditions
}

\author{
Malte Asseln ${ }^{1 *}$, Patrick Meere ${ }^{2}$, Peter Walker ${ }^{2}$, Klaus Radermacher ${ }^{1}$ \\ ${ }^{1 *}$ Chair of Medical Engineering, Helmholtz Institute for Biomedical Engineering, RWTH Aachen \\ University, Aachen, 52074, Germany \\ ${ }^{2}$ Department of Orthopaedic Surgery, New York University Hospital for Joint Diseases, New \\ York, 10003, USA \\ asseln@hia.rwth-aachen.de
}

\section{Introduction}

An important factor which influences the clinical outcome of total knee arthroplasty (TKA) is the internal/external rotational alignment of the femoral and tibial components. A rotational error of few degrees has been found to be clinically important and linked to anterior knee pain [1] and patellofemoral problems [2]. A commonly used reference to align the tibial component rotationally is the tibial tuberosity (TT). However, Howell et al. [3] showed that the medial/lateral TT position varies strongly between patients and represents an unreliable landmark during (passive) kinematic component alignment. Thereby, passive kinematics differs from active kinematics [4,5]. For example, under weight-bearing conditions the magnitude of internal/external rotation is greater and occurs earlier [6]. In the active situation, a relative internal rotation of the tibial component increases the Q-angle with a change in the force vector of the extensor mechanism.

An established radiographic measure to quantify the relative rotational alignment of the femur and tibia in extension is the tibial tuberosity-trochlear groove (TT-TG) distance. It has been measured recently at different flexion angles under non-weight-bearing conditions [7]. However, Izadpanah et al. [8] reported that it also depends on weight-bearing. Hence, the complex function of the extensor mechanism in terms of the relationship between TT position, TT-TG distance, and internal/external rotation under weight-bearing conditions is still unknown and accordingly component rotational alignment targets.

The aim of the study was to investigate the relationship between TT position, TT-TG distance, and internal/external rotation of the knee in the active (weight-bearing) situation. 


\section{Materials and Methods}

The study was based on 8 healthy subjects (age: $30-50$ years, gender: 8 males, body height: 1.68 $1.91 \mathrm{~m}$, body weight: $67-112 \mathrm{~kg}$ ). The geometry data and in vivo weight-bearing kinematics of a single legged stance were acquired in a previous study by our group [9]. High-resolution magnetic resonance imaging (MRI) data in supine position had been registered with low-resolution quasi-static MRI data in loaded positions at 5 different knee flexion angles. Three-dimensional surface geometries of the knee were obtained from manual segmentation of the high-resolution MRI data. The TT attachment was approximated by the mean of the insertion area and identified in the MRI data by a clinical expert. Anatomical axes for each bone were defined based on anatomical references. The proximal/distal axis of the femur and tibia were aligned along the most superior centroid of the femoral shaft and the most inferior centroid of the tibial shaft, respectively.

In a proprietary MATLAB program (Mathworks Inc., USA) the femoral geometries were transformed into the tibial anatomical coordinate system for the flexion angles and the TT-TG distance was measured following the description by Schoettle et al. [10]. Therefore, the vertices of the femoral polygon mesh were projected into the tibial anatomical transversal plane (Figure 1). Only the first 4 flexion angles were considered, because the posterior condylar axis could not be identified properly in high flexion angles.

In addition, the relative medial/lateral position of the TT was calculated by dividing its distance to the medial tibial border by the overall mediolateral width. Thus, its position was expressed as percentage of the mediolateral distance (\%ML). Joint kinematics, particularly internal/external rotation, was calculated using the established "floating axis" system. The relationship between the parameters was investigated by using linear correlation analysis in MATLAB.

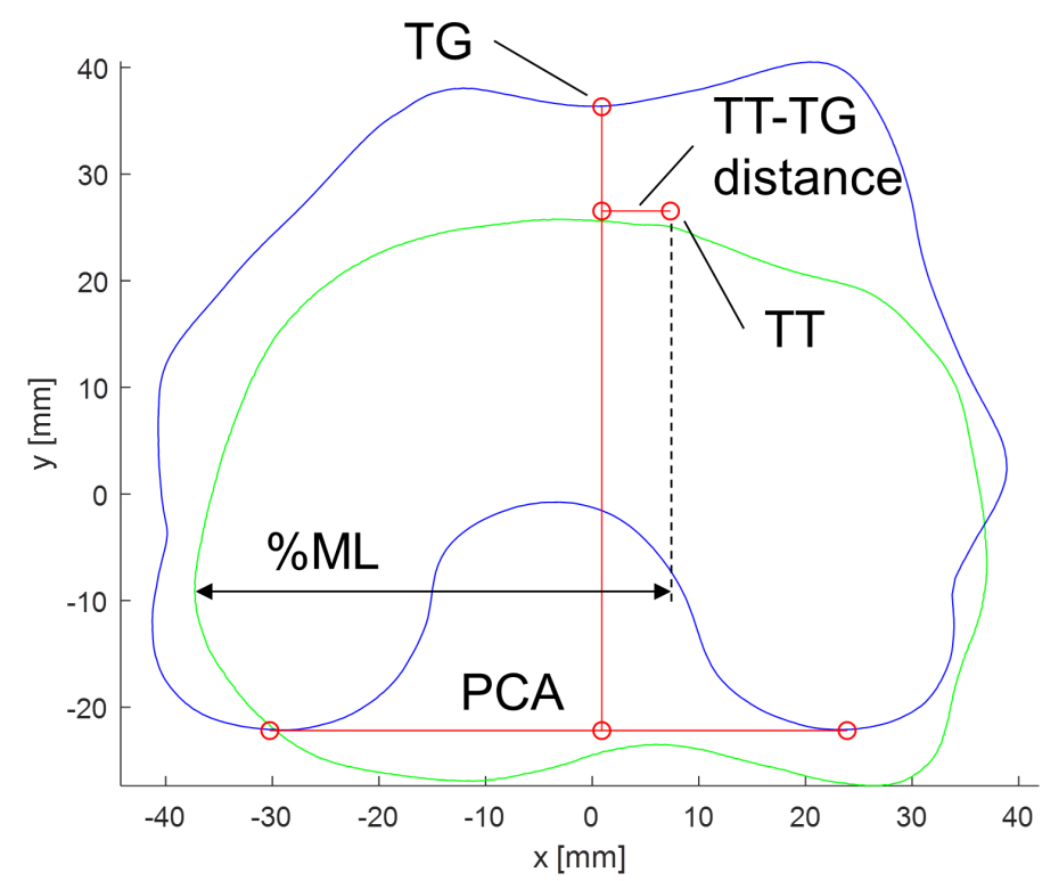

Figure 1: Measurement of the TT position and TT-TG distance in overlaid projections in the tibial anatomical transversal plane $($ TG $=$ Trochlear Groove, TT $=$ Tibial Tuberosity, PCA = Posterior Condylar Axis, $\% \mathrm{ML}=$ relative distance to medial tibial border). 


\section{Results}

The result of the TT-TG distance over knee flexion is presented in Figure 2 (A). There was an overall tendency of a decrease with greater knee flexion. The mean TT position was $0.61 \pm 0.04 \% \mathrm{ML}$ (ranging from $0.55-0.66 \% \mathrm{ML}$ ) meaning that all subjects showed a lateralized TT position. The linear correlation between initial TT-TG distance and TT position is visualized in Figure 2 (B). The results showed a good correlation $\left(\mathrm{R}^{2}=0.7730\right)$ and indicate that a greater TT-TG distance is associated with an increased lateral TT position. If this is true, a lateralized TT position is not compensated by an internal rotation of the tibia or external rotation of the femur, respectively. Thus, it was questioned, whether a lateral TT position results in an increased internal/external rotation under weight-bearing conditions with M. quadriceps activation from extension to mid-flexion, as suggested by our earlier in-silico study [11]. The kinematic results are presented in Figure 2 (C). A good correlation was observed for 6 of 8 subjects $\left(R^{2}=0.7709\right)$ (Figure $\left.2(D)\right)$. In these cases, a greater lateral TT position resulted in an increased internal rotation of the tibia between extension and mid-flexion $\left(20-25^{\circ}\right)$. The other 2 subjects (S02, S03) did not fit into the scheme. However, they showed the overall lowest internal/external rotation of the entire cohort.

(A)

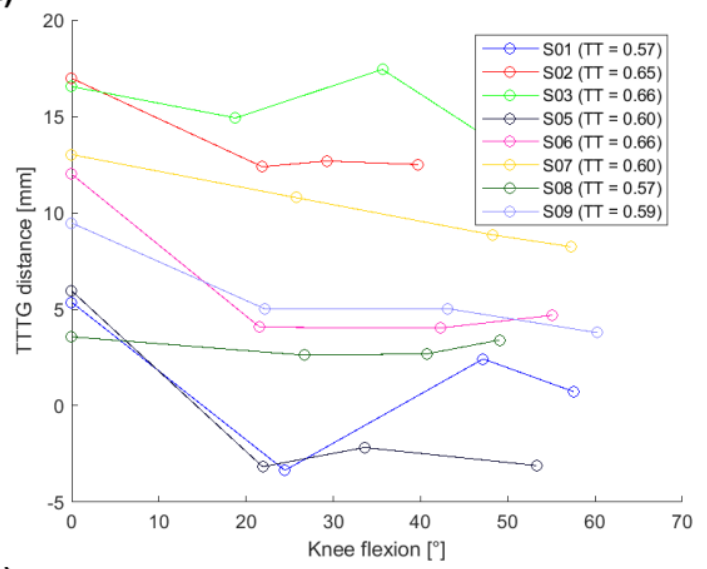

(C)

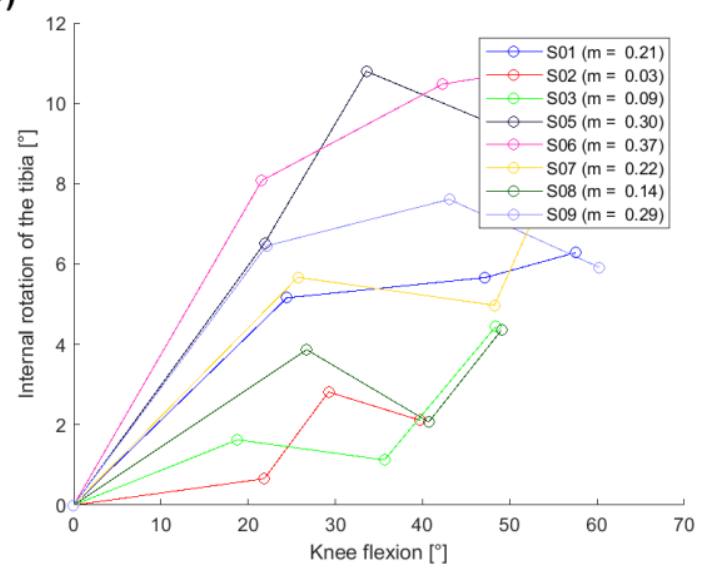

(B)

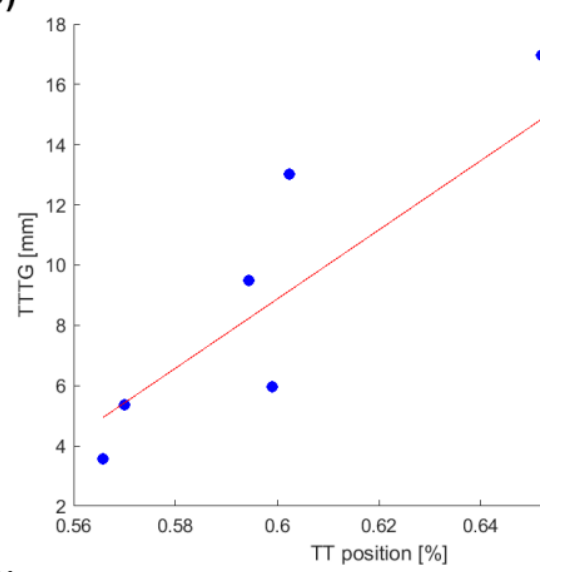

(D)

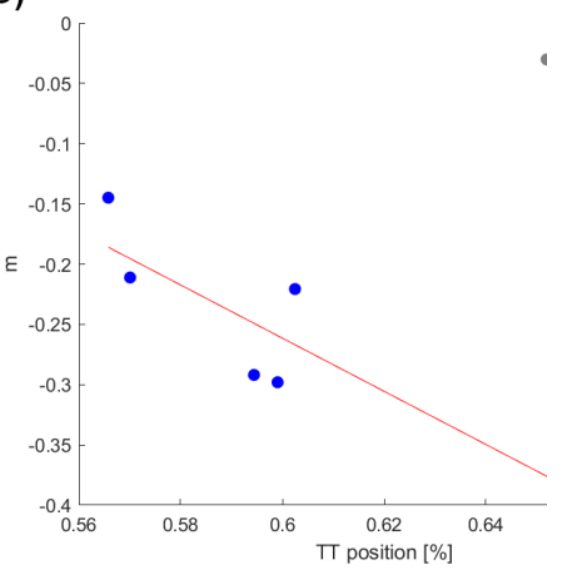

Figure 2: (A) TT-TG distance at different knee flexion angles. (B) Correlation between TT-TG distance and TT position. (C) Internal rotation of the tibia at different knee flexion angles $(\mathrm{m}=$ slope from the line from extension to the first upright MRI position). (D) Correlation between TT position and slope $\mathrm{m}$. 


\section{Discussion}

In this study, we investigated the relationship between TT position, TT-TG distance, and internal/external rotation under weight-bearing conditions. The TT-TG distance values ranged between $5-17 \mathrm{~mm}$ in extension, which is considered as being normal [12,13]. Our results showed an overall tendency of decreased TT-TG distance values over knee flexion, which coincides with previous findings [8,7]. The medial/lateral TT position and TT-TG distance showed a good correlation in extension (Figure 2 (B)), which have not been reported previously to the best of our knowledge. This finding is of importance since it indicates that in extension, where M. quadriceps is rather inactive, a lateralized TT position is not compensated by an internal rotation of the tibia. Looking at the internal/external rotation 6 of 8 subjects showed a good correlation between TT position and internal rotation of the tibia. The greater the lateral TT position, the higher the internal rotation during flexion. The other 2 subjects fall out of the series, which might be explained by compensatory mechanisms, such as patellar kinematics, J-Curves, tibial slopes, etc. which is part of future investigations. In conclusion, the position of the TT and TT-TG distance are functionally relevant in the weight-bearing kinematics and should be carefully considered in preoperative TKA planning and intraoperatively to avoid any disharmony and conflicts in joint mechanism.

\section{References}

[1] Barrack RL, Schrader T, Bertot AJ, Wolfe MW, Myers L. Component rotation and anterior knee pain after total knee arthroplasty. Clinical orthopaedics and related research 2001:46-55.

[2] Berger RA, Crossett LS, Jacobs JJ, Rubash HE. Malrotation causing patellofemoral complications after total knee arthroplasty. Clinical orthopaedics and related research 1998:14453.

[3] Howell SM, Chen J, Hull ML. Variability of the location of the tibial tubercle affects the rotational alignment of the tibial component in kinematically aligned total knee arthroplasty. Knee surgery, sports traumatology, arthroscopy official journal of the ESSKA 2013;21:228895.

[4] Dennis DA, Komistek RD, Stiehl JB, Walker SA, Dennis KN. Range of motion after total knee arthroplasty: the effect of implant design and weight-bearing conditions. The Journal of arthroplasty 1998;13:748-52.

[5] Draper CE, Besier TF, Fredericson M, Santos JM, Beaupre GS, Delp SL et al. Differences in patellofemoral kinematics between weight-bearing and non-weight-bearing conditions in patients with patellofemoral pain. Journal of orthopaedic research official publication of the Orthopaedic Research Society 2011;29:312-7.

[6] Johal P, Williams A, Wragg P, Hunt D, Gedroyc W. Tibio-femoral movement in the living knee. A study of weight bearing and non-weight bearing knee kinematics using 'interventional' MRI. Journal of biomechanics 2005;38:269-76.

[7] Suomalainen J-S, Regalado G, Joukainen A, Kääriäinen T, Könönen M, Manninen H et al. Effects of knee flexion and extension on the tibial tuberosity-trochlear groove (TT-TG) distance in adolescents. Journal of experimental orthopaedics 2018;5:31.

[8] Izadpanah K, Weitzel E, Vicari M, Hennig J, Weigel M, Südkamp NP et al. Influence of knee flexion angle and weight bearing on the Tibial Tuberosity-Trochlear Groove (TTTG) distance 
for evaluation of patellofemoral alignment. Knee surgery, sports traumatology, arthroscopy official journal of the ESSKA 2014;22:2655-61.

[9] Al Hares G, Eschweiler J, Radermacher K. Combined magnetic resonance imaging approach for the assessment of in vivo knee joint kinematics under full weight-bearing conditions. Proceedings of the Institution of Mechanical Engineers. Part H, Journal of engineering in medicine 2015;229:439-51.

[10] Schoettle PB, Zanetti M, Seifert B, Pfirrmann CWA, Fucentese SF, Romero J. The tibial tuberosity-trochlear groove distance; a comparative study between CT and MRI scanning. The Knee 2006;13:26-31.

[11] Asseln M, Zimmermann F, Eschweiler J, Radermacher K. The Q-angle and its effect on active kinematics - a simulation study. In: Proceedings of the 13th Annual Meeting of the International Society for Computer Assisted Orthopaedic Surgery; 2013, p. 193-195.

[12] Dejour H, Walch G, Nove-Josserand L, Guier C. Factors of patellar instability: An anatomic radiographic study. Knee Surg, Sports traumatol, Arthroscopy 1994;2:19-26.

[13] Sobhanardekani M, Sobhan MR, Nafisi Moghadam R, Nabavinejad S, Razavi Ratki SK. The Normal Value of Tibial Tubercle Trochlear Groove Distance in Patients With Normal Knee Examinations Using MRI. Acta medica Iranica 2017;55:573-7. 